Published as: Deeb, R., Ooms, K. \& De Maeyer, P., 2011, Typography in the Eyes of Bertin, Gender and Expertise Variation. Cartographic Journal, 49 (2), 176185.

\title{
Typography in the Eyes of Bertin, Gender and Expertise Variation
}

\author{
Rasha Deeb, Kristien Ooms, Philippe De Maeyer \\ Gent University, Department of Geography \\ Krijgslaan 281, S8, B-9000, Gent, Belgium \\ \{Rasha.Deeb, Kristien.Ooms, Philippe.DeMaeyer\}@UGent.be
}

\begin{abstract}
The main goal of this research is to set a group of typographic criteria to suit a wide array of map users. A group of Bertin's visual variables were applied individually and associatively for the same set of labels. Two kinds of maps (with point and areal objects) were presented to expert and non-expert map users and analysed accordingly. Additionally, the effect of gender variation was taken into account. The data were aggregated and studied for each graphical variable. For some combinations of Bertin's variables, statistically significant differences were detected in the preferences of the different map users (e.g., male vs. female and expert vs. non-expert). Consequently, we identified which graphical variables (individually or combined) were more preferred by specific user groups in relation to their application on text objects.
\end{abstract}

Keywords: Bertin's variables. User preference. Text variations.

\section{BACKGROUND AND OBJECTIVES}

Reading a map is a task that cannot be accomplished unless textual elements are provided. These texts, and more specifically the labels on the map image, can be considered the fourth symbol type in addition to points, lines and areas. This finding implies that certain rules should be applied in the typographic design (Fairbairn, 1993). 
Board and Taylor (1976) demonstrated the advantages of using a conventional symbology and its influence on reading names. Gerber (1981) identified reading labels as a midterm level of the reading process, while Robinson and Petchenik (1976) defined the reading as an active process and a transaction between the individual and the environment. Therefore, it is important to know how much information is transferred to each reader.

Variations in how labels are presented on the map (e.g., size and colour value to indicate a hierarchy) facilitate the interpretation of the contents (Imhof, 1975). Bartz (1970a) also examined the influence of font size and its adequate dimension. When used incorrectly, however, the variations in label presentation may influence the interpretation of the map's contents in a negative way. Therefore, it is necessary to know which type of variation can be used on map labels to allow efficient interpretation.

Bertin (1970) defined six visual variables (size, value, texture, colour, orientation and shape) that are applied to the symbols on a map to visualise properties of objects or links between objects, including associativity, selectivity, order and quantity. In Table 1 the links between the six visual variables and their properties are illustrated.

Table 1 Levels of organization of the visual variables. After Bertin (1970, fig. 2, p.69)

\begin{tabular}{|c|c|c|c|c|}
\hline Visual variable & Associativity & Selectivity & Order & Quantity \\
\hline Size & & & & \\
\hline Value & & & & \\
\hline Texture & & & & \\
\hline Colour & & & & \\
\hline Orientation & & & & \\
\hline Shape & & & & \\
\hline
\end{tabular}

Text can be considered the fourth symbol type, therefore these visual variables should also be applicable in this case (Fairbairn, 1993). Text objects on a map do not have the same design rules as point, line and area objects. It is distinct in the way visual variables are applied. The 'translation' of the application of these visual variables to text symbol type is described below and illustrated in Table 2:

- $\quad \underline{\text { Size }}$ is applied to text by changing the font size, consuming more space on the map for larger corpus and less space for smaller corpus. In this article, points (dots) are used to indicate texts sizes. Boldness was added to this variable as the word consumes more space for the same font size. Size provides selective, ordered and quantitative perception. Therefore, variations in size can indicate quantitive information like the population of a city, but this can also be used to order information (discrete classes indicating population levels) using levels of hierarchy among the different label classes.

- Value is defined by Bertin (1970) as the ratio between black and white perceived on a given surface. Changing the value of the typographical variable serves order and selectivity, but it is still a critical concern when the printing method is used (gray tone or coloured), in addition to the background of the map. Based on the variation in value different label levels with hierarchal purposes are served.

- Texture (or pattern) is represented by a combination of differing text characteristics. The texture of typographical symbols is linked with the overall layout of the individual 
word, such as the narrow or wide spacing of letters. In addition to that, texture is recognized by the overall view of all textual elements on the map image, reflecting the relationship of labelled features. Size and value work together to provide infinitive array of textures. Texture is an associative variable that allows users to identify variations and group correspondences within all depicted categories. Selectivity and order can also be visualised using this variable. These properties can thus be used to indicate a distinct themes (e.g. continent vs. ocean) or certain label hierarchy (e.g. city vs. village).

- Colour (or hue) of texts is the attribute of the visual sensation on a fixed saturation and value. Colours provide both an associative and a selective perception. Text colour can work functionally and is usually employed to differentiate between themes, such as blue for water bodies and red for danger.

- Orientation can be applied to text either on each individual character (italic), or by tilting the whole word. Orientation plays an important role in the associative and selective perception. Unlike in books, texts on maps can follow many orientations to stress the shape of the labelled object, such as rivers or countries.

- Shape is represented using different fonts. 'The world of shape is infinite' Bertin said, and this holds also true for text objects with an unlimited number of fonts. Associativity is the property that is obtained by using different shapes, which allows the map reader to distinguish between different categories and group similar objects. Consequently, shape can describe different themes at the same level of importance. This is commonly used when labelling, for example, land use maps.

Table 2: Bertin's variables on text

\begin{tabular}{|l|l|l|l|}
\hline \multicolumn{1}{|c|}{ Variable } & \multicolumn{3}{|c|}{ Examples } \\
\hline \multirow{2}{*}{ Size } & Cartography & Cartography & Cartography \\
\cline { 2 - 4 } & Cartography & Cartography & Cartography \\
\hline Value & Cartography & Cartography & Cartography \\
\hline $\begin{array}{l}\text { Texture } \\
\text { within a word }\end{array}$ & Cartography & Cartography & Cartogl|aphly \\
\hline $\begin{array}{l}\text { Texture } \\
\text { in a group } \\
\text { of words }\end{array}$ & $\begin{array}{l}\text { CARTOGRAPHY } \\
\text { Cartography } \\
\text { cartography }\end{array}$ & $\begin{array}{l}\text { CARTOGRAPHY } \\
\text { Cartography } \\
\text { cartography }\end{array}$ & $\begin{array}{l}\text { Cartography } \\
\text { Cartography } \\
\text { Cartography }\end{array}$ \\
\hline Colour & Cartography(red) & Cartography(green) & Cartography(blue) \\
\hline Shape & Cartography & Cartography & Cartography \\
\hline
\end{tabular}

After Bertin defined these visual variables and their properties, some authors took a closer look at them in the light of new psychological theories or cartographic development. Imhof (1975) added the variable position which he also applied to texts as a label can be placed at a number of (fixed) locations relative to its associated objects. He described the four preference positions of labels associated with point objects as the upper right corner, lower right corner, upper left corner and lower left corner. The investigation of the optimal position for labels, however, is not discussed in this paper. MacEachren (1995) thoroughly discussed 
the visual variables based on their $\mathrm{X}, \mathrm{Y}$ dimensions together with their numerical, ordinal and nominal applications. In addition to the dimensions of the symbology, modern cartography also allowed the development of new variables, such as sound (MacEachren, 1994) and time (Kraak et al., 1997). Koch (2001) referred to Bertin's variables as an application of the Gestalt laws, which describe rules of similarity and good design. These laws have been extended after Bertin, but they are still valid in the modern cartography. For $2 \frac{1}{2} \mathrm{D}$ and $3 \mathrm{D}$ representations Slocum et al. (2005) rephrased Bertin's 2D variables using spacing, size, perspective height, orientation, shape, arrangement and finally hue, lightness and saturation.

The legibility of maps has been a concern of cartographers since they started developing cartographic products. Additionally, maps are widely used products by many different groups of users. Consequently, text as a distinct component requires special care in the long-term development of maps. Locating names based on typographic variations and measuring the completion times of tasks was explored by Bartz (1970b), who conducted a series of experiments in which participants had to locate names on a map. This gave insights in the efficiency of certain typographic variables towards the map user. Foster and Kirkland (1971) studied the association between text and different colours. Phillips et al. (1977) compared the response times of users related to different text characteristics, such as size, boldness and shape, for difficult and easy names. Kraak and Ormeling (2010) defined rules for texts on maps to improve their readability. They also suggested utilising different textual variables to produce a more readable map that also meets the needs of the text functions.

The studies mentioned above investigated the legibility of labels using the amount of time a map user needs to complete a task. However, none of these studies considered the combination of all Bertin's variables and their implementation on the textual information on maps as well as their influence on the legibility of the map. These studies also used paper maps to examine a range of individual text variables and fixation time measurement to indicate the best text visualisation. Furthermore, no link was made from the application of Bertin's variables to texts. This paper aims to extend these studies by measuring user preferences when applying Bertin's variables to texts (excluding colour and value), taking into account experience and gender as each of these groups may respond differently to maps.

When designing a map, different choices have to be made: type of map, theme of the map, map audience, use of the map, etc. Two of the most important types of maps are topographic and thematic maps. The way the labels are placed and visualised on these maps greatly depends on their type, theme and audience. In the study described in this paper, two basic map types are used: maps with point data and maps with areal data which are all labelled. The first category (with point data) can refer to topographic maps, isopleths maps and dot maps whereas the second category (areal data) can refer to the majority of qualitative and quantitative thematical maps such as choropleth Maps, soil maps, socio-cultural maps and socio-economical maps. A blank background maps are used as a basic of extensive studies where more complex map design will be tested and colour and value will be tackled.

The experiment is a user study in which the participant must choose one of two maps that have variations in how labels are visualised (cf. Bertin's variables). Since different types of users are included in the study (experts, non-experts, females, males), the outcome of the study reveals whether cartographic training and practice influences label preference on maps. 
This method allows differentiating the variables used on maps according to who the map intended for. The objective of the experiment is to answer the following questions:

- What size does the audience prefer in its ratio to the general space of the map?

- What is the influence of bold fonts on the audience's preference?

- What kind of fonts does the user tend to accept more?

- Which aspect of the orientation variable do the audience prefer the most?

- What is the influence of simple label textures and complex label textures on users' preference?

- Do all users have the same preferences when there are variations in the level of experience in map use and in gender?

- How different levels of label hierarchy can be presented using Bertin's variables and what is the impact of these designs on users' preference?

\section{THE METHODOLOGY}

An experiment was constructed to examine the users' preference towards the application of Bertin's visual variables to texts for both point and areal data. The study provides an overview of map users' preferences of labelling characteristics based on variation in experience and gender.

\section{Participants}

A group of 80 map users participated in the study. They were divided according to the criteria experience and gender. The non-expert group included 50 participants in the beginning of their geographical education, with no previous training in cartography. The other group of participants consisted of 30 experts who work with maps on a daily basis and have at least a master's degree in geography. Of the 80 participants, 35 were female and 45 male divided in balance across experts (15 females and 15 males) whereas the non-expert group consisted of 20 females and 30 males. The average age of the users was 23.3 years, with the experts' average on 27.3 years and the non-experts on 20.4 years. By taking into account experience and gender, it was possible to detect what influence the users' backgrounds had on their preferences regarding labels on maps.

\section{Task, stimuli and data}

Forty-one maps were presented to the users during the experiment in pairs or triples, forming 79 sets of questions. Base maps were drawn at the scale of 1:100 000 ${ }^{1}$. These maps were designed with a blank background in order to acquire neutral results of users' preference. The study had both a between- and within-user design. The first part of the experiment involved maps populated with point data and their associated labels. The graphical variables size, shape and texture were applied to the map labels to visualise levels of importance in the labels and thus in the associated objects. An example of such a pair of maps is depicted in Figure 1.

\footnotetext{
${ }^{1}$ The dimension of the test structure changed the scale to 1:270000.
} 
During the second part of the experiment, variations in the size, shape and orientation of labels associated with areal objects were investigated. Two maps from these experiments are illustrated in Figure 2. Bertin's variables were applied (individually and combined) and integrated in a coherent structure for both series. Colour and value were excluded from these stimuli because the function of these variables is mainly to indicate the nature of the visualized object (Kraak and Ormeling ,2010) which is not the domain of this research. In addition to that, text colour and value cannot work functionally with a blank background because of the consistent interaction between the text foreground and the background's colours.

To avoid biases in the answers due to resolution and size differences, all participants completed their test on a flat screen with a 1280x1024 resolution. Each participant followed the same order of maps in a sequence that lasted 20-30 minutes.

These trials were embedded in an online questionnaire in which two or three maps appeared on the screen simultaneously. The participant then indicated a ranking, which was subsequently stored in a database. The result was an ordered list of the maps for each participant, indicating his preferences on the readability of the maps for the different visualised graphical variables.

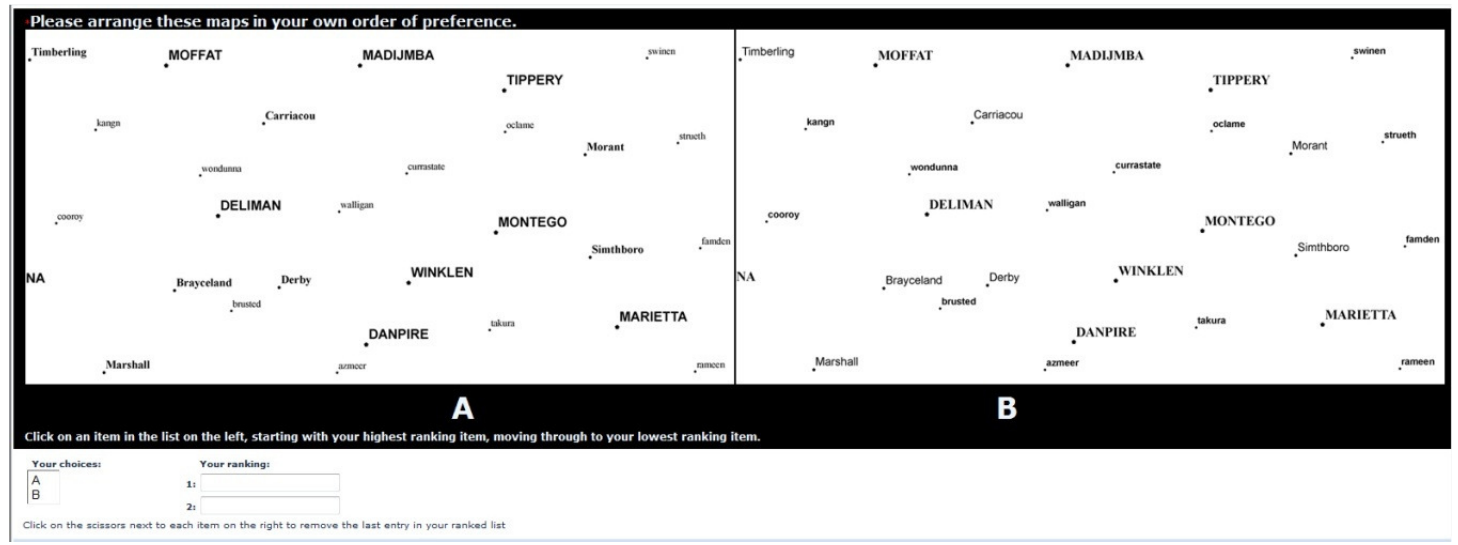

Figure 1: Example of the ranking task for point data (variable: texture)

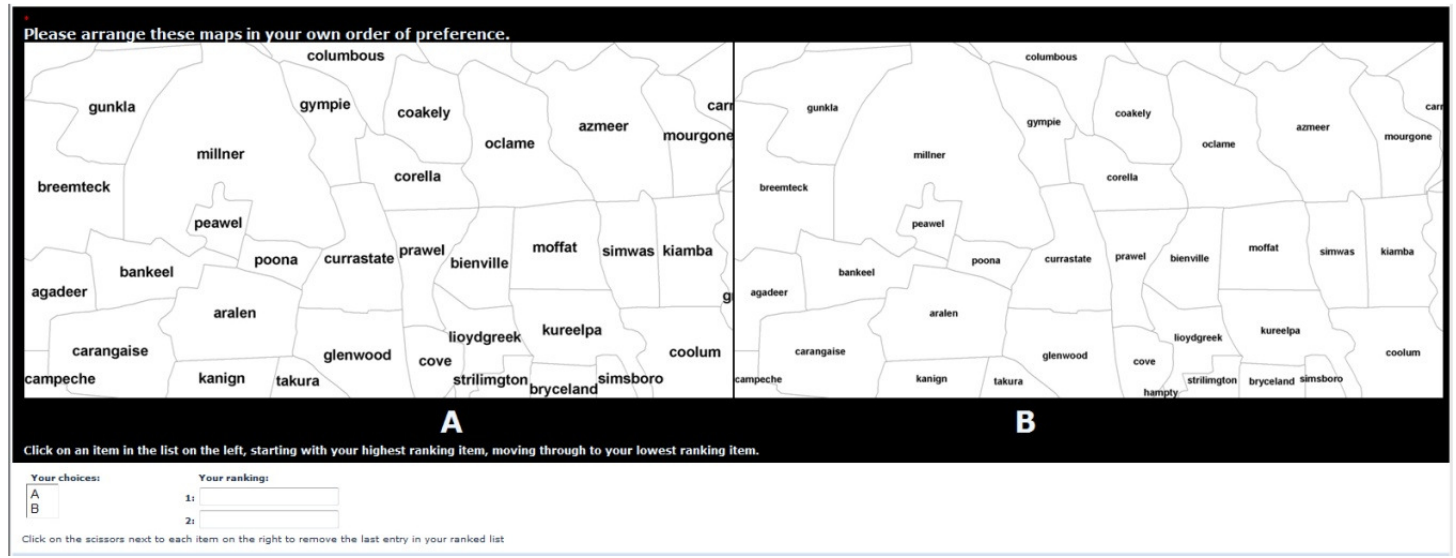

Figure 2: Example of the ranking task for areal data (variable: size) 


\section{Measurement of preference and typographic quality for all users}

Map aesthetics is an important aspect of cartographic work. To recall organised cartographic symbols factually and sensibly, care should be taken in choosing the adequate symbols for a wide range of audiences. Therefore, a variety of typographic symbols were presented to the map audience to test which presentation was preferred. The collected data were accumulated and restructured to indicate the preferable criteria between each set of maps.

A selection of criteria was used to obtain the most preferred quality that measures aesthesis. Typographic criteria were analytically organised and grouped in order to reflect four major mapping purposes:

- The equivalent use of criteria of names and labels.

- The hierarchal use of criteria of names and labels.

- The thematical use of criteria.

- A combination of the above purposes, for example to emphasize certain elements.

Thanks to the combination possibilities of visual variables, numerous functionality can be expressed using different level of complexity. One of the most important functions is the hierarchical importance which can be embedded in three level of complexity. Complexity refers to the systematic combination of the visual variables. Accordingly, the labels (and maps) used in this study were divided into three levels of complexity:

- First level of complexity: one of Bertin's visual variable was assigned to labels and text (e.g showing hierarchy using only different font sizes).

- Second level of complexity: two variables were combined or labels were presented in a hierarchical way using a visual variable in addition to font size.

- Third level of complexity: formed by using more than two associated variables. Complex hierarchy is formed by adding tow visual variables to font size which can be link to it to form different hierarchal textures (case styles, boldness, etc.).

\section{Size}

Regarding the first level of complexity, size as a typographical variable varied from 8- to 14font size. Due to the fact that the map's dimensions were transferred according to the test structure; the sizes used were called A, B, C and $\mathrm{D}^{2}$. These sizes were compared pair wise and summed to reveal which font size was preferred. The results, listed in Table 3, show the order of preference to be B-A-C-D. Although font size measurement was changed due to map scale changes, the users' preference was calculated for the overall view of map and the ratio of label size to the area size. When only considering the labels' font sizes, the largest size (D) showed the least interest from the users and consequently, it did not acquire a high rate of aesthetics evaluation. Here, the label size $\mathrm{D}$ is considered too big relative to area they cover. Size $C$ is also rather large but it has a noticeable higher preference rate than $D$. The smallest size did not match the highest preference rate compared to size B which is the most preferred size. Consequently, this reflects the highest aesthetics design by the symmetry and harmony

\footnotetext{
${ }^{2} \mathrm{~A}$ corresponds to the original size $8, \mathrm{~B}$ to $10, \mathrm{C}$ to 12 , D to 14 . The scaling factor applied to each map when implementing it into the questionnaire is 0.37 . The original 8 point size is transferred to 2.96 points, 10 points become 3.7 points, 12 points become 4.44 points and 14 points is transferred to 5.18 points.
} 
between the label font size and the district size. This result demonstrates the relation between the labels to their depicted area.

Table 3: Font size overall preference

\begin{tabular}{cccccc}
\hline font size & A & B & C & D & total \\
\hline A & 0 & 33 & 49 & 62 & 144 \\
B & 47 & 0 & 60 & 74 & 181 \\
C & 20 & 31 & 0 & 71 & 122 \\
D & 9 & 6 & 18 & 0 & 33 \\
\hline
\end{tabular}

The second level of complexity was also related to the size of the labels; however, in this case, bold and uppercase characters were used to indicate hierarchy. The variations in the case styles were presented using 'all letters in lowercase', 'only first letter in uppercase', 'all letters in upper case'. These levels of hierarchy were both tested with a serif (Times New Roman, TNR) and sans serif (Arial) font. The results are illustrated in Table 4. These variations in case styles and boldness show a higher preference for the bold size in comparison to not bold for all forms of case style. The general ratio is about $60 \%$ bold preference to $40 \%$ not bold preference and this result remains valid for a sans serif font (Arial) and a serif font (Times New Roman). The results also show that the case style has a slight deference regarding both used font (Arial, TNR).

Table 4: Boldness effect on text preference

\begin{tabular}{ccccc}
\hline Font & Size & $\begin{array}{c}\text { All letters are } \\
\text { lowercase }\end{array}$ & $\begin{array}{c}\text { First letter is } \\
\text { uppercase }\end{array}$ & $\begin{array}{c}\text { All letters are } \\
\text { uppercase }\end{array}$ \\
\hline \multirow{2}{*}{ Arial } & bold & 49 & 42 & 49 \\
& not bold & 31 & 38 & 31 \\
\multirow{2}{*}{ Times New Roman } & bold & 45 & 45 & 47 \\
& not bold & 35 & 35 & 33 \\
\hline
\end{tabular}

On the third level of complexity, size was obtained by varying case styles combined with other visual variables to present multiple levels of hierarchy (two or three levels). Complexity overlaps with hierarchy when different levels of hierarchy need to be presented. For such a level of complexity, three sequential font sizes correspond to three levels of importance. Here the highest level was completely uppercase, the middle level was designed with first letter uppercase and the lowest was fully written in lowercase letters (= 3 levels). This was later compared with a map containing labels with only two levels of hierarchy $(=2$ levels); as the highest level was fully designed in uppercase and the other two were both labelled with first letter uppercase. These results are presented in Table 5. The simple hierarchy (2 levels) shows $20 \%$ more preferences than the one with 3 levels for an Arial font and $40 \%$ more preference for Times New Roman. By comparing the results of the levels of complexity and the difference between Arial and Times New Roman, the users' preference shows the importance of simplicity when designing labels.

Table 5: The third level of complexity preference (with 2 and 3 levels of hierarchy)

\begin{tabular}{|c|c|c|c|c|}
\hline \multirow{2}{*}{ Hierarchy } & \multicolumn{2}{|c|}{ Arial } & \multicolumn{2}{|c|}{ Times New Roman } \\
\hline & 2 levels & 3 levels & 2 levels & 3 levels \\
\hline bold & 48 & 32 & 56 & 24 \\
\hline not bold & 44 & 36 & 56 & 24 \\
\hline
\end{tabular}




\section{Shape}

Shape preference was evaluated based on the frequency that participants chose for each studied shape. Different fonts were used to test shape preference. Since the number of shapes (fonts) is theoretically infinite, 15 different shapes were defined and divided into 5 groups (related to formality, spacing, serif, serif and spacing, sans serif and spacing) each with three levels. Each group thus tests one characteristic of shape which ranged from a low over middle to a high level. Figure 3 illustrates the user preference of each characteristic. This shows that users prefer fonts that have the moderate degree of formality, spacing and serif and prefer the lowest degree of serif and spacing and sans serif and spacing. When presenting fonts characteristics in a ranked order, it should be noted that the lowest level is preferred when two criteria of font are presented and the middle characteristic when un individual criterion is presented.

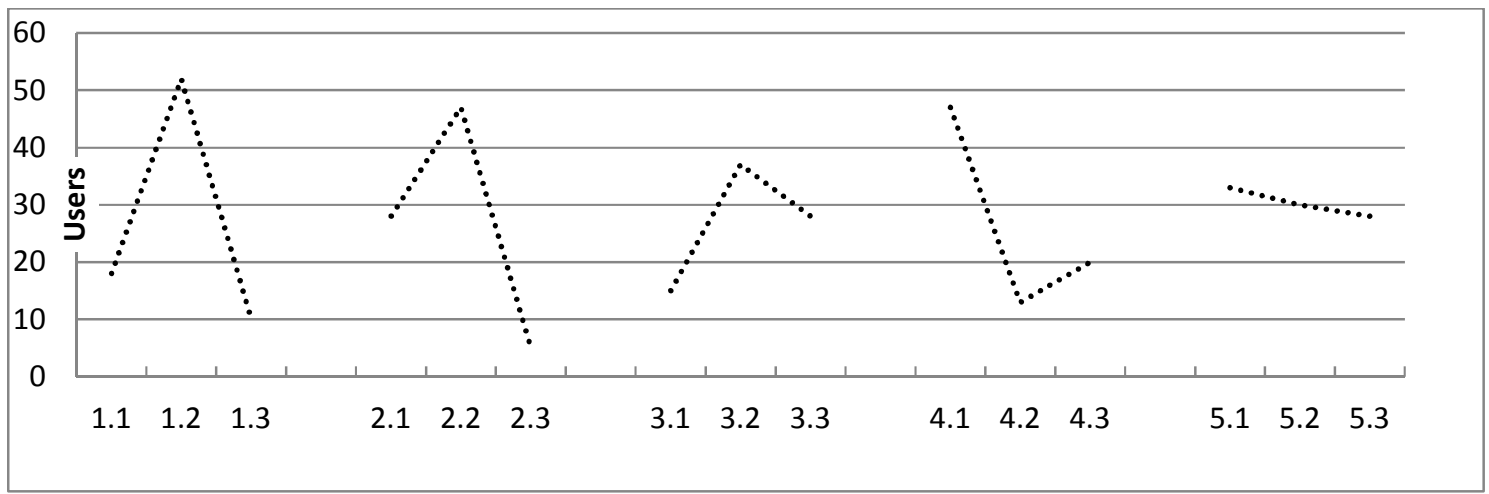

\begin{tabular}{|c|c|c|c|c|c|c|c|c|c|}
\hline fantasy & 1.1 & spacing 0 & 2.1 & serif 0 & 3.1 & serif \& spacing 0 & 4.1 & sans serif \& spacing 0 & 5.1 \\
\hline formal & 1.2 & spacing 1 & 2.2 & serif 1 & 3.2 & serif \& spacing 1 & 4.2 & sans serif \& spacing 1 & 5.2 \\
\hline historical & 1.3 & spacing 2 & 2.3 & serif 2 & 3.3 & serif \& spacing 2 & 4.3 & sans serif \& spacing 2 & 5.3 \\
\hline
\end{tabular}

Figure 3: Shape preference of the same texture

Three methods of labelling according to case style were tested for the two most frequently used types (Times New Roman and Arial). Upper case, lower case and first letter upper case labelled map were tested pair wise. The arguable theory of serif and sans serif use of typography was thus tested. Table 6 represents the users' shape preference showing the highest preference for Arial with about $70-80 \%$ vs. a $20-30 \%$ preference for Times New Roman.

Table 6: The influence of shape on text preference

\begin{tabular}{ccc}
\hline Case style & Times New Roman & Arial \\
\hline All letters lower case & 20 & 60 \\
First letters upper case & 27 & 53 \\
All letters upper case & 15 & 65 \\
\hline
\end{tabular}

\section{Orientation}

The orientation of typographic symbols was studied in two phases. First, the orientation of the entire word was evaluated by the participant. The orientation of the labels used during the study corresponds to a typical output of most cartographical software: all horizontal, all tilted (under the angle of the largest diagonal) or mixed (horizontal if applicable within the boundaries, otherwise tilted). The participants tended to prefer the horizontal orientation, 
followed by the tilted orientation and finally the mixed type orientation. This is listed in Table 7. Under the circumstances of the experiment, it was found that the users' preference of horizontal matches the orientation of text reading.

Table 7: Orientation influence on text preference

\begin{tabular}{lcc}
\hline Orientation & Participants & $\%$ \\
\hline Horizontal & 59 & 74 \\
Tilted & 10 & 12 \\
Mixed & 11 & 14 \\
\hline
\end{tabular}

In the second phase, the orientation of each letter was considered. The italic style was tested with the three levels of complexity (see Table 8). At the first level of complexity, straight and italic was tested for four font sizes of the labels (A, B, C, D). The straight orientation was preferred over all the tested sizes (A, B, C, D). The second level of complexity involved italic vs. bold italic in pair wise comparison. In this case, only the smallest size acquired the highest preference for bold italic, while the opposite is true for italic (with also a rather high value for medium sizes). For the third level of complexity, the result is exactly the opposite. Bold italic was tested vs. narrow bold italic in this case. The smallest font $\mathrm{A}$ has the highest preference for bold italic whereas the preference of narrow bold italic is for the larger font sizes B, C, D. It is important to note that the users' preference of second and third level of complexity is thus dependent on font size.

Table 8: Complexity effect on orientation of text preference

\begin{tabular}{ccccccc}
\hline & \multicolumn{2}{c}{$\begin{array}{c}\text { First level of } \\
\text { complexity }\end{array}$} & \multicolumn{2}{c}{$\begin{array}{c}\text { Second level of } \\
\text { complexity }\end{array}$} & \multicolumn{2}{c}{$\begin{array}{c}\text { Third level of } \\
\text { complexity }\end{array}$} \\
Font size & Straight & Italic & Italic & Bold italic & Bold italic & Narrow bold italic \\
\hline A & 65 & 15 & 17 & 63 & 64 & 16 \\
B & 42 & 38 & 47 & 33 & 38 & 42 \\
C & 47 & 33 & 47 & 33 & 17 & 63 \\
D & 74 & 6 & 70 & 10 & 9 & 71 \\
\hline
\end{tabular}

\section{Texture}

The design of texture has a wide array of choices as they are based on a combination of visual variables. As a consequence, the textures were divided according to font type: serif (Times New Roman) and sans serif (Arial). These two main groups were further subdivided according to the applied variables: textures of the same font and same size, multi-texture of the same font and different sizes and finally multi-texture of multi-font and different sizes. For each mentioned division the users identified which one they preferred, which is shown in Figure 4. Using one way ANOVA, the test declared that no statistical significant deference was detected for the three groups of texture and between the two font types $(F=0.00$, $P=1.00)$. However, when only one font was considered, the texture designed of Arial was more preferred. In addition to that, the textures designed of bold fonts were more preferred. 


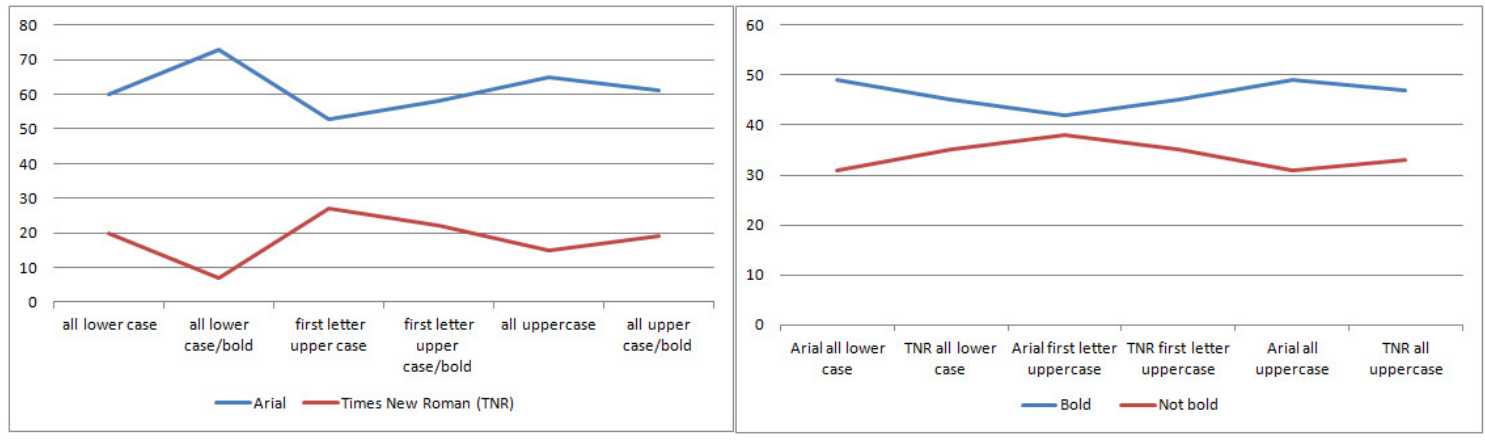

Figure 4: The preference of shape and size of different textures

\section{Experience and gender influence}

Both clusters of experience and gender were tested by asking participants to show their preference of 79 sets of two or three map combinations. These sets are devoted to test size, shape, orientation and texture. For each set users' preference was analysed statistically using two-sided T-test.

\section{Experts vs. non-experts}

Expert participants are well trained to use maps and work with maps on a daily basis. It was expected that their attitude towards map symbols, especially the typography, would therefore differ from the non-expert users. The ranking test was presented to both user groups (experts and non-experts). Shown in Table 9, using 2 sided T-test only three statistical significant different were found. They are emerged in the variables of orientation, shape and texture.

Table 9: The detected level of preference variation between experts and non-experts

\begin{tabular}{lcc}
\hline CRITERIA (Experts/ Non-experts) & Pearson Chi-Square & Asymp. Sig. (2-sided) \\
\hline Arial-narrow-B size-bold vs. Arial-narrow-B size-bold-italic & 3.63 & 0.049 \\
Arial-bold-all uppercase vs. TNR-bold-all uppercase & 7.735 & 0.005 \\
Arial-not bold-2 levels vs. TNR-not bold-2 levels & 4.832 & 0.028 \\
\hline
\end{tabular}

Figure 5 illustrates these significant differences between the experts' and non-experts' preference. The variation between the two groups occurred in the sub-case of orientation Arial narrow bold italic B size. The test showed a fair significant difference (0.049). This draws attention to the care that should be taken in labelling with B size as the most preferable and readable size. The second significant difference was the same texture (all letters were in uppercases), although with a different font. A high significant difference was recorded (0.005) as the experts preferred Arial font type for this task, whereas the non-experts preferred Times New Roman (TNR). This difference between a serif and a sans serif font must be taken into account when creating maps for different user groups. Similarly, the same texture with two levels of hierarchy and different bold fonts showed a high significant difference (0.028), with a majority preferring Arial. 


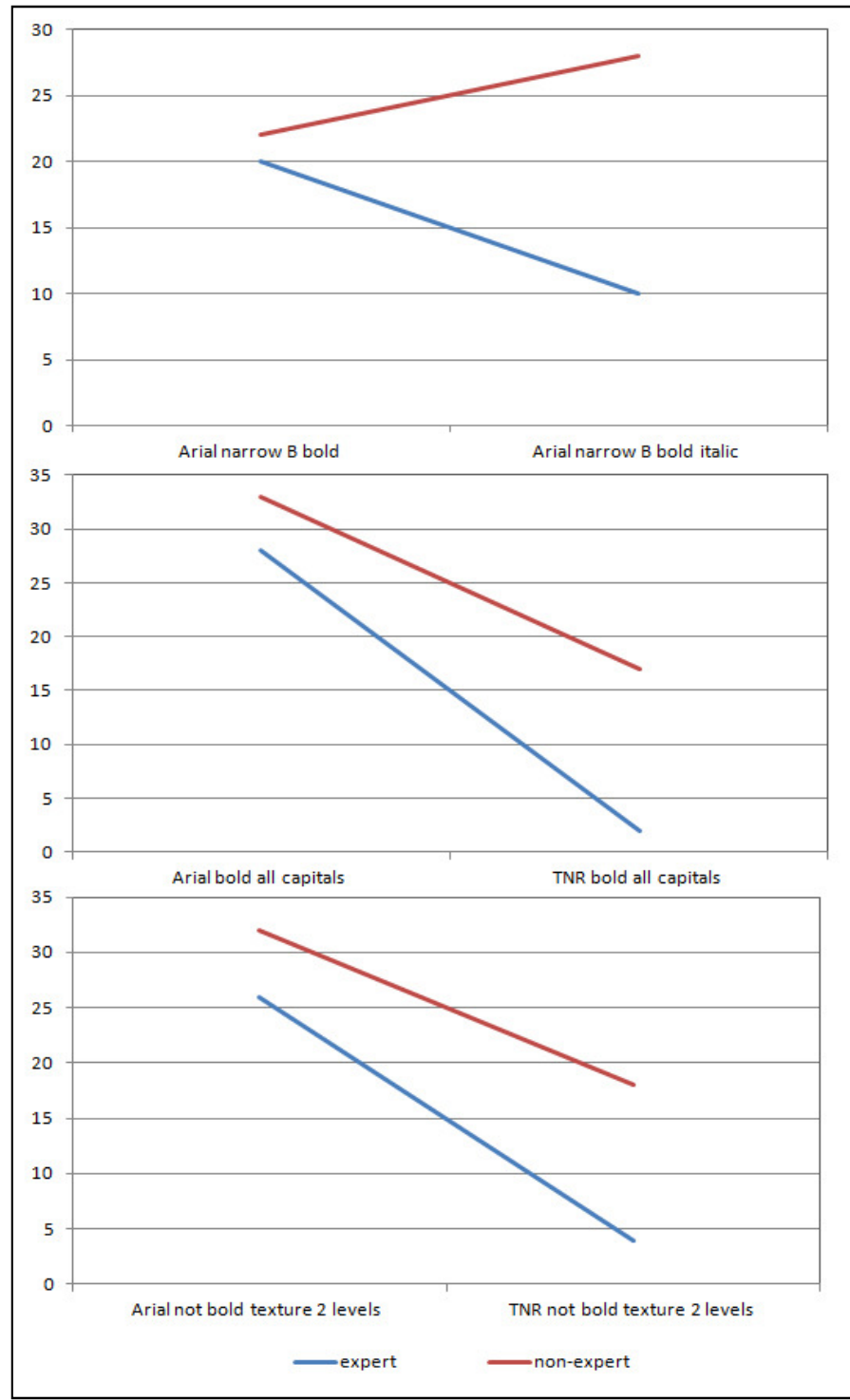

Figure 5: Significant differences between experts and non-experts

\section{Females vs. males}

Concerning the preferences of females and males, data were compared pair wise between the two user groups. Four significant differences were recorded when using T-test (see Table 10). Starting from size B bold versus D bold, a high significant deference was located (0.025) as females rejected the larger size and preferred the B size. This case was recorded only for the bold size, which in turn demands much more attention and care for the association with other variables. The second significant difference was located between Arial narrow $\mathrm{C}$ bold and Arial narrow $\mathrm{C}$ bold italic (0.042). The third significant difference (0.047) was detected for the uppercase which enlarges the symbols and makes the details more visible, in addition to the difference between Arial and Times New Roman (TNR). The final significant difference regards the same texture and different font of Arial and Times New Roman was highly significant (0.005). The texture used for this test was a combination of the three levels of 
hierarchy and boldness for size. The result was a decreased preference for Times New Roman by female participants.

Table 10: The detected significance levels for gender preference variations

\begin{tabular}{lcc}
\hline \multicolumn{1}{c}{ CRITERIA (Females/Males) } & Pearson Chi-Square Value & Asymp. Sig. (2- \\
\hline Arial-D size-bold vs. Arial-B size-bold & 5.045 & 0.025 \\
Arial-narrow-C size-bold vs. Arial-narrow-C size-bold-italic & 4.127 & 0.042 \\
Arial-all uppercase vs. TNR- all uppercase & 3.940 & 0.047 \\
Arial-bold- 3 levels vs. TNR-bold-3 levels & 8.061 & 0.005 \\
\hline
\end{tabular}
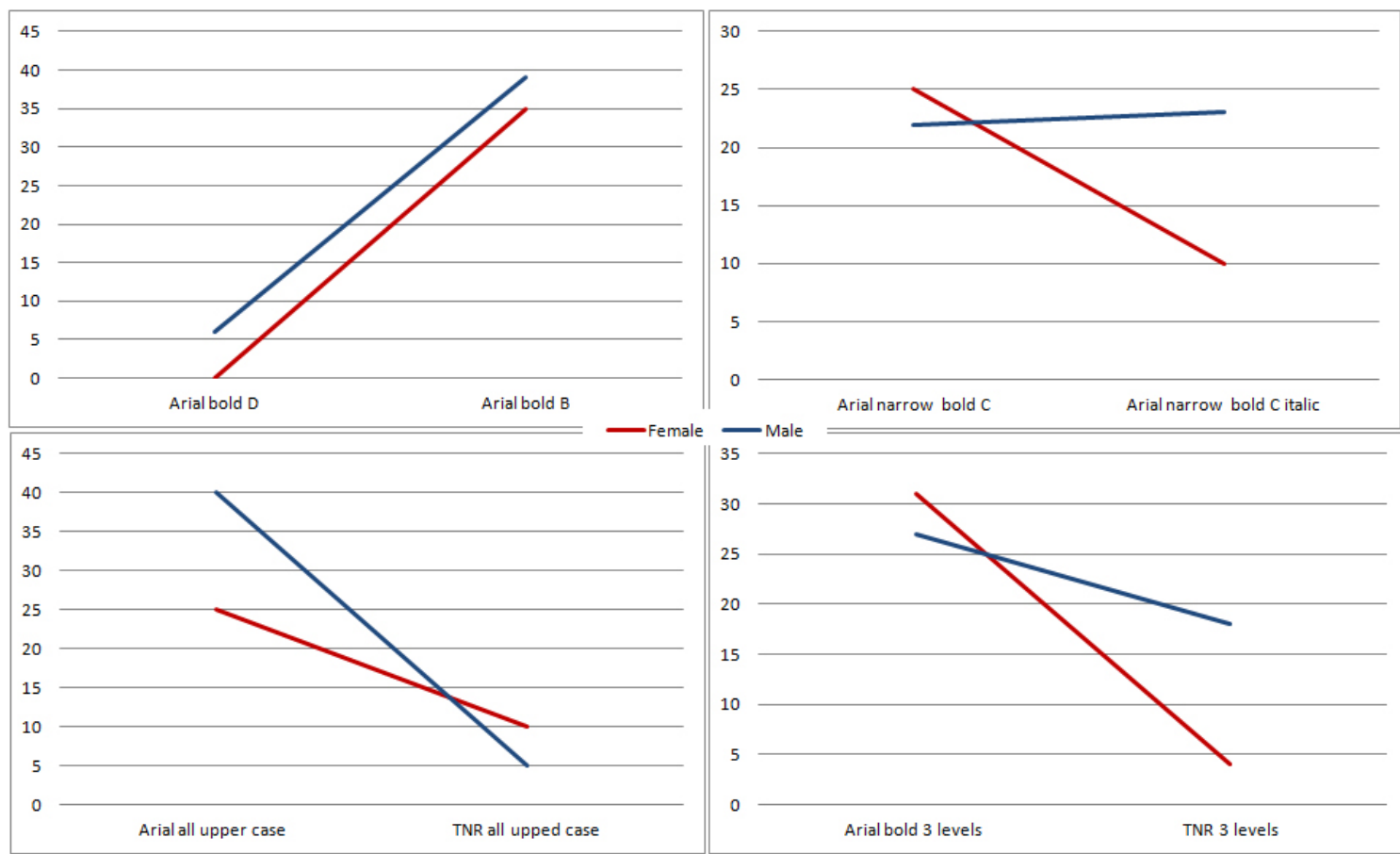

Arial all upper case

Arial bold 3 levels

TNR 3 levels

Figure 6: Located significant differences for females and males

\section{CONCLUSION AND FUTURE PERSPECTIVES}

It is not possible to define one typographic variable as the 'best' variable for all typographic functions and for all map users. Although it has been found that some criteria have much more acceptance than others, it is still unclear which is the most efficient and legible. To acquire these insights, further experiments are required with time measurement as a scale of efficiency.

The levels of complexity utilised declared few remarkable differences for some criteria, such as narrow typography, italic and bold. Additionally, contradictions were found when using complex textures compared to font size. This finding is due to the fact that boldness makes the symbol larger and narrower. It has also been concluded that when size is combined to other variables such as narrow and italic the larger size is highly preferred over the smaller. 
For the three levels of complexity, different typographic criteria are the most preferable and legible for the examined group. Bertin's preferred variable on typography can be classified as follows:

- According to the map scale used and the distribution of labels illustrated previously in Figures 1 and 2 (including point data and area data), font size B is most preferred by participants working with screen maps.

- A bold font allowed the typographic symbols to stand out more, thus making it more eye-catching for the participant. A higher level of preference for bold fonts, as illustrated in Figure 4, confirms this finding. In maps of a higher complexity, however, bold fonts tended to be a detrimental variable.

- Arial is the most preferred font type as an individual variable. This finding could limit some cartographic design. However, this result is not only true when the font is varied individually; but also when font (shape) is associated with other variables such as size and texture, Arial type is generally preferred.

- It is remarkable that the preference of different shapes followed almost the same trend in the analyses of both experience and gender. Text simplicity or complexity represented by font showed no specific significant difference, meaning that the preference trend for each group could be explained by the overall trend of shape preference and vice versa.

- Horizontal orientation is the most preferable typographical variable. However, it has the disadvantage of assigning the orientation of the geographical features. Additionally, the text might exceed the available space for a small feature size. According to the cartographical rules and needs, map designers cannot always utilise horizontal orientation.

- The orientation of the word letters (straight vs. italic) varies depending on the level of complexity design. For the first level of complexity, users show noticeable preference of the straight orientation mean while it was remarkably noted that for the second and third level of complexity the user preference depend on the labels font size.

- For point data labelling, it is critical to establish rules as it has been shown that differing groups have varying tendencies, especially when it comes to the second and third levels of complexity.

- The results indicated some statistical difference within the experience and gender clusters. What is remarkable is that these differences were not about variables of the first level of complexity but about the second and third one.

- The visual variables of order perception (size, value and texture) can be used individually or combined to introduce hierarchy. This research presented hierarchy by ordered variation in size (font size, boldness and case style). Using two levels of hierarchy was remarkably preferred over using three levels of hierarchy. This result remains valid for Arial (sans serif) and TNR (serif) fonts. 
The preferences of the examined groups unsystematically vary between them and according to the studied variable, which requires more standardising techniques and other studies to acquire more detailed insights in the legibility and efficiency of typographic symbols. Additionally, the results are only valid on a blank background where the distribution of features is the most important function of the map and thus the primary function of text is to provide the geospatial address. Obtaining more empirical information on the relationship between labels and the surrounding map elements is essential and will be included in further studies, such as different background colours.

However, care should be taken when studying colour and value. They are very critical variable because of the interaction between the colour of labels and the colours of map elements in first place. Secondly, the medium on which the map will be presented is critical as well. Therefore, a thorough study is planned to obtain insights in the relation between label visualisation and the map background (topographic, thematic and their use of colours): which value is the best for a certain layout, the influence of colour onto map legibility and others.

Determining the best legible typographic variable is a combination of two tasks, starting with map aesthesis and ending with map efficiency, including a series of rules and priorities regarding map design. Further studies need to be undertaken in order to explain the relation between text functions and their design, which plays a critical role for utilising different variables individually or combined.

Furthermore, it would be interesting to extend this work to the applications of label on other language symbols than Latin in order to test the influence of visual variable on different lettering systems such as Chinese or Arabic. Moreover, the efficiency of visual variable on typography will be tackled in future publication.

\section{REFERENCES}

Bratz, B.S. (1970) 'An Analysis of the Typographic Legibility Literature, Assessment of Its Applicability to Cartography', The Cartographic Journal, 7(1), pp. 6-17.

Bratz, B.S. (1970) 'Experimental Use of The Search Task in The Analysis of Type Legibility in Cartography', The Cartographic Journal, 7(2), pp. 103-113.

Bertin, J. (1970) Semiology of Graphics: Diagram, Networks, Maps, Translated by William J. Berg, Esri press, California.

Board, C. And Taylor, R.M. (1976) 'Perception and Maps: Human Factors in Map Design and Interpretation', Transactions of the Institute of British Geographers, 2(1), pp. 19-36.

Fairbairn, D.J. (1993) 'On The Nature of Cartographic Text', The Cartographic Journal, 30(2), pp. 104-111.

Foster, J.J and Kirkland, W. (1971) 'Experimental studies of map typography', Bulletin of the society of university cartographers. 6(1), pp. 40-45.

Gerber, R. (1981) 'Competence and performance in cartographic language', The Cartographic Journal. 18 (2), pp. 104-111.

Imhof, E. (1975) 'Positioning Names on Maps', The American Cartographer, 2(2), pp. 128144.

Koch, W.G . (2001) 'Jacques Bertin's Theory of Graphics and Its Development and Influence on Multimedia Cartography', Information Design Journal, 10(1), pp. 37-43. 
Kraak, M.J and Ormeling, F. (2010) Cartography. Visualization of Spatial Data, $3^{\text {rd }}$ edition, Person Prentic Hall, Harlow.

Kraak, M.J., Edsall R. and MacEachren, A.M. (1997) "Cartographic Animation and Legends for Temporal Maps: Exploration and or Interaction", Proceedings 18th ICA/ACI International Cartographic Conference, Stockholm, Sweden.

MacEachren. A.M and Taylor. D.R.F. (1994) Visualization in Modern Cartography, Pergamon, Oxford.

MacEachren. A.M.(1995) How Maps Work, Representation, Visualization and Design, the Guilford press, New York.

Phillips, R.J.,Noyes.L \& Audley.R.J. (1977) 'The Legibility of Type on Maps', Ergonomics, 20(6), pp. 671-682.

Robinson, A. H. and Petchenik , B.B. (1976) The Nature of Maps, University of Chicago press, Chicago.

Slocum.T.R, McMaster.R.B, Kessler.E.C, Haward.H.H. (2005) Thematic Cartography and Geographic Visualization, $2^{\text {nd }}$ edition, Person Prentic Hall, Upper Saddle River, NJ. 